\title{
GREEN SYNTHESIS OF SILVER NANOPARTICLES USING POLYSACCHARIDE EXTRACTED FROM LAURENCIA OBTUSA ALGAE
}

Magdy K. Zahran and Hana A. Mohammed

Chemistry Department, Faculty of Science, Helwan University, Ain-Helwan, Cairo, 11795, Egypt.

Key Words: silver nanopartecles, polysaccharide, L. obtusa, FT-IR, XRD and TEM

\section{ABSTRACT}

The aim of this work was to synthesis of silver nanoparticles (AgNPs) using water soluble Polysaccharide extracted from marine algae (L. obtusa) as reducing agents for silver ions as well as stabilizing agents for the synthesized AgNPs. The formation of silver nanoparticles was confirmed by Surface Plasmon Resonance (SPR) at 350 to $550 \mathrm{~mm}$, by using fourier transform infrared spectroscopy (FT-IR), X-ray diffraction (XRD) and transmission electron microscopy (TEM). The maximum absorption peaks are in the range of $400-420 \mathrm{~nm}$. The particles were spherical in shape in the range of $4-10 \mathrm{~nm}$.

\section{INTRODUCTION}

Many researchers have widely used noble nanoparticles in various technological applications because of their unique properties. The noble metal nanoparticles, in general, and silver nanoparticles (AgNPs), in particular, are known for their versatile applications in medical [Becker, 1999], food processing [Tankhiwale and Bajpai, 2010] and textile industries [Duran $\boldsymbol{e t}$ al., 2010] as well as in consumer goods [Jiang $\boldsymbol{e t}$ al., 2004; Rai et al., 2009].

A large number of physical, chemical, biological, and hybrid methods are available to synthesize different types of nanoparticles [Liu et al., 2001]. The nanoparticles formed using each method show specific properties. However, biosynthesis of metal nanoparticles by plant extracts is a green modern alternative for their production [Shameli $\boldsymbol{e t}$ al., 2012]. Green synthesis of nanoparticles makes use of environmentally friendly, non-toxic and safe reagents [Salam et al., 2012].

The main objectives of the present study are: (i) to synthesize AgNPs using polysaccharide of $L$. obtusa algae (ii) to characterize AgNPs using UV-Vis spectroscopy, FT-IR, XRD and TEM.

\subsection{Material}

\section{EXPERIMENTAL}

The red algal used throughout the present work were collected from the beach of Red Sea, Alexandria, Egypt. These were found attached to 
the rocky belt existed about 15 meters from the sea-shore and at about two meters depth and were Freshly collected algal species were repeatedly washed with sea-water followed by tap-water to remove sand, salt and any extraneous matters. The cleaned samples were shade dried and homogenized to fine powder and kept for further treatments.

\subsection{Extraction of polysaccharide}

The defatted algae powder was successively percolated with hot water till complete exhaustion. The extract was concentrated to about 50 $\mathrm{ml}$ under reduced pressure using rotary evaporator device. Polysaccharide were precipitated using Absolute ethanol $(250 \mathrm{ml})$ which added dropwise with stirring till complete precipitation occurred. The residue obtained was washed with absolute ethanol then weighed and saved for further study.

\subsection{Synthesis of silver nanoparticles (AgNPs)}

AgNPs were synthesized from silver nitrate using polysaccharide of $L$. obtusa as reducing and stabilizing agent. $30 \mathrm{mg}$ of the extract residue dissolve $95 \mathrm{ml}$ of distilled water and adjust $\mathrm{pH}=12$ using sodium Hydroxide and complete volume to $99 \mathrm{ml}$ with distilled water then put it on magnetic stirrer hot plate at temperature $90{ }^{\circ} \mathrm{C}$ and added $1 \mathrm{ml}$ of (0.1M) $\mathrm{AgNO}_{3}$ solution were added to the reaction mixture then the reaction mixture was kept at different durations $(15,30,45$, and 60min).

\subsubsection{Ultraviolet-visible (UV-vis) spectroscopy analysis}

Change in color was visually observed in the silver nitrate solution incubated with polysaccharide of L. obtusa. The reduction of silver ions was monitored from 300 to $800 \mathrm{~nm}$ by Jasco V-670 UV-V after 5-fold diluting the sample with distilled water against distilled extract as blank at a resolution of $1 \mathrm{~nm}$.

\subsubsection{Fourier transform infrared (FTIR) spectroscopy analysis}

For FTIR spectroscopy measurements, the bio-synthesized AgNPs were purified, dried and palleted with potassium bromide in the ratio of 1:100. FTIR spectrum of samples was recorded on (JASCO FT-IR 4100 instrument, Japan). All measurements were carried out in the range of $400-4000 \mathrm{~cm}^{-1}$ at a resolution of $4 \mathrm{~cm}^{-1}$.

\subsubsection{Transmission electron microscopy (TEM).}

The size and morphology of the synthesized AgNPs were determined by high resolution transmission electron microscopy (TEM, JEOL JEM 2100, Japan). The sample for TEM studies was prepared as follows: $1 \mathrm{ml}$ of the reaction mixture containing AgNPs was diluted to $5 \mathrm{ml}$, sonicated using ultrasonic bath and a 
drop of it was placed on a $\mathrm{Cu}$ grid with ultrathin $\mathrm{Cu}$ on holey $\mathrm{C}$ film and it was allowed to dry in a vacuum. The instrument was operated with an acceleration voltage of $200 \mathrm{kV}$.

\subsubsection{X-ray diffraction (XRD)}

The synthesized nanoparticles were examined by XRD (6000 shimadzu - Japan). The powdered AgNPs was stacked in the cubes of XRD and the result was taken in the XRD equipment at this condition: $\mathrm{X}$-ray tube target, copper potassium alpha radiation; voltage, $40.0(\mathrm{kV})$; current, 30.0 (mA). Divergence slit, 1.00000 (deg); scatter slit, 0.00000 (deg); receiving slit, $0.30000(\mathrm{~mm})$. Scanning drive axis, Theta-2Theta, scan range, 4.0000 - 90.0000 (deg); scan mode, continuous scan; scan speed, 8.0000 (deg/min); sampling pitch, 0.0200 (deg).

\subsection{Visible observation}

\section{RESULTS AND DISCUSSION}

The Colour of L. obtusa Polysaccharide before addition of $\mathrm{AgNO}_{3}$ solution is yellow, after its treatment with $\mathrm{AgNO}_{3}$ solution, the colour changes to reddish-brown which indicated the formation of AgNPs (Fig. 1). This colour change is due to surface Plasmon excitation of the formed nanoparticles which affects the optical property.

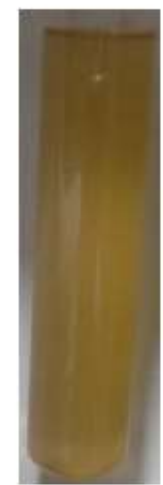

A

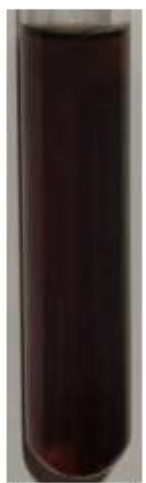

B

Figure 1: Colour change before and after addition of $\mathrm{AgNO}_{3}$ solution. Where (A) the polysaccharide of L. obtusa algae (B) the biosynthesized AgNPs

\subsection{UV-vis spectra analysis}

$\mathrm{UV}-\mathrm{V}$ is absorption spectroscopy is an important technique to determine the formation and stabilization of biosynthesized AgNPs in aqueous solution. AgNPs were synthesized at optimized 
conditions of temperature $90^{\circ} \mathrm{C}$ and $\mathrm{pH}=12$ meanwhile the time was varied $(15,30,45$ and $60 \mathrm{~min})$, the formation of silver nanoparticles was monitored by UV spectrophotometer. The maximum absorption peaks are in the range of $400-420 \mathrm{~nm}$ which is a typical plasmon band of silver metal [Durán et al., 2005; Ahmad et al., 2003], suggesting the formation of silver nanoparticles (Fig. 2).

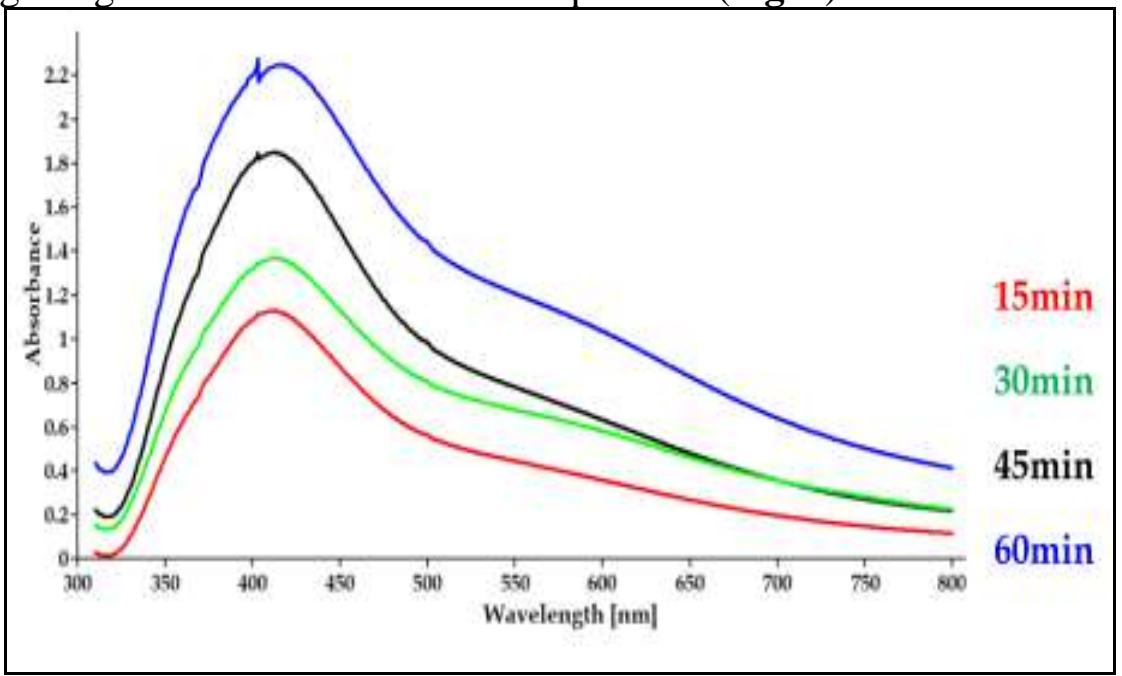

Figure 2: UV-Visible absorption spectra of biosynthesized silver nanoparticles from polysaccharide extract of L. obtusa at different times interval, constant $\mathrm{pH}$ and temperature.

\subsection{FTIR spectroscopy}

FTIR study reveals the multi-functionality of $L$. obtusa polysaccharide by identifying the possible functional groups in the biomolecules of this poly saccharides. A FT-IR spectrum of the synthesized AgNPs and polysaccharide by this green method is shown in Figure 3. Data of this figure indicate that the polysaccharide manifest absorption peaks at about 3441 were assigned to $\mathrm{OH}$ stretching vibration, the peak at $2932 \mathrm{~cm}^{-1}$ corresponds to $\mathrm{C}-\mathrm{H}$ alkane, 1480 represent $-\mathrm{C}=\mathrm{C}-$ group. The peak at $1141 \mathrm{~cm}^{-1}$ corresponds to $\mathrm{C}-\mathrm{O}$ stretching vibration. These results were previously proved by Parida et al., 2014; Namvar et al., 2014. As is evident (Fig. 3), both FTIR spectra of the Polysaccharide extract and the Polysaccharide extract containing AgNPs have the same trend but the intensity of functional groups present in nanoparticlrs sample is small, and this proves that the different functional groups in the bioactive compounds polyphenols, protein and polysaccharide are consumed for reduction of $\mathrm{Ag}^{+}$to $\mathrm{Ag}^{0}$ and stabilization of the biosynthesized AgNPs. 


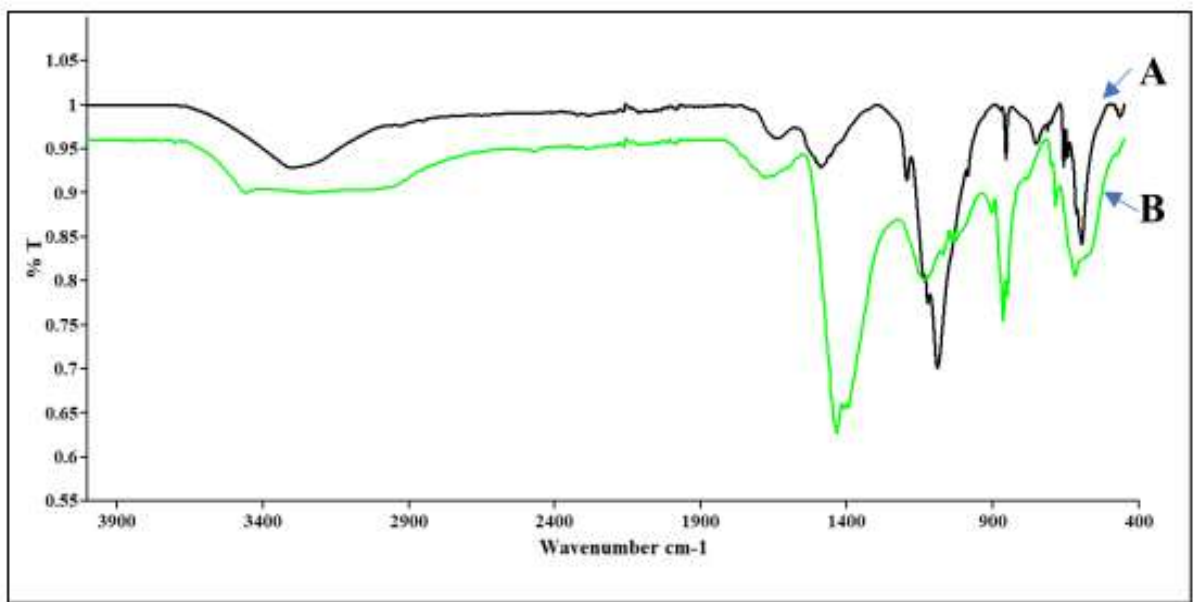

Figure 3. FTIR spectra of (A) Polysaccharide extract of L. obtusa algae and (B) biosynthesized AgNPs

\subsection{Transmission Electron Microscope (TEM)}

TEM provides further insight into the morphology and particle size distribution profile of the AgNPs and revealed a pattern similar to that of the biosynthesized AgNPs characterized by TEM [Bindhu and Umadevi, 2013; Das et al., 2013]. The data obtained from transmission electronmicrograph (Fig. 4) showed distinct shape and size of AgNPs. The particles were spherical in shape in the range of 5-10 nm.

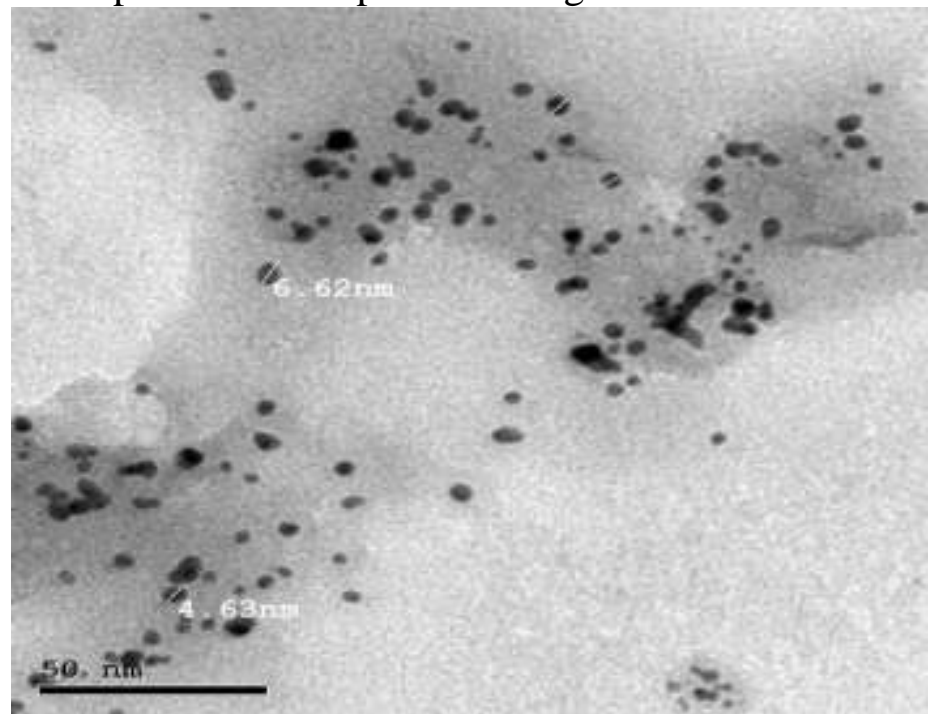

Figure 4. TEM of AgNPs from polysaccharide of L. obtusa algae. 


\subsection{XRD analysis}

Analysis through X-ray diffraction was carried out to confirm the crystalline nature of the particles, and the XRD pattern showed numbers of Braggs reflections that may be indexed on the basis of the face centered cubic ( $\mathrm{fcc}$ ) structure of silver. A comparison of our XRD spectrum with the standard confirmed that the silver particles formed in our experiments were in the form of nanocrystals, as evidenced by the peaks at $2 \theta$ values of $38,44.7$, and 65 , and $77.7 \theta$, corresponding to (111), (200), (220) and (311), respectively Bragg reflections of silver. The XRD results clearly show that the silver nanoparticles formed by the reduction of $\mathrm{Ag}^{+}$ions by the L. obtusa polysaccharide are crystalline in nature [Umoren et al., 2014].

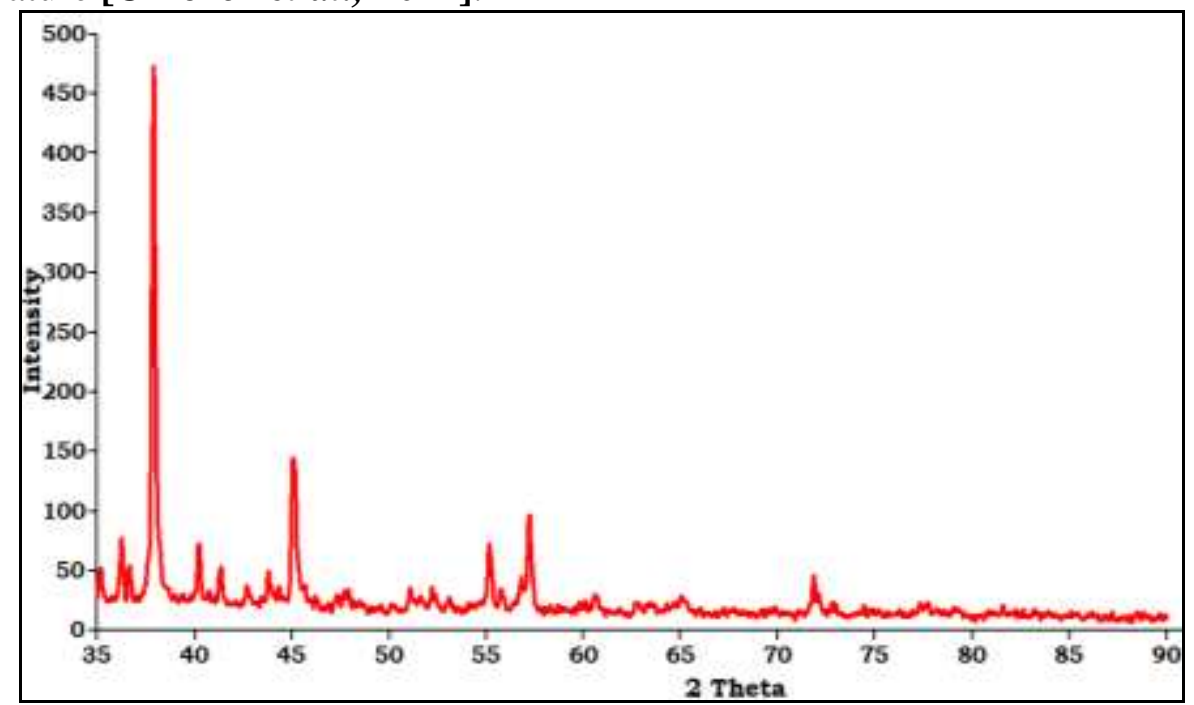

Figure 5. XRD pattern of AgNPs from Polysaccharide of L. obtusa algae .

\section{CONCLUSION}

Green chemistry approach towards the synthesis of nanoparticles has many advantages such as, ease with which the process can be scaled up and economic viability. We have developed a fast, eco-friendly and convenient method for the synthesis of silver nanoparticles using $L$. obtusa polysaccharide with a diameter range of size $5-10 \mathrm{~nm}$. These particles and spherical. No chemical reagent or surfactant template was required in this method, which consequently enables the bioprocess with the advantage of being environmentally friendly. Color change occurs due to surface plasmon resonance (SPR) during the reaction with the ingredients present in the polysaccharide extract results in the formation of silver nanoparticles which is confirmed by UV-Vis, FT-IR, TEM and XRD. 


\section{REFERENCES}

Ahmad, A.; P. Mukherjee ; S. Senapati ; D. Mandal ; M.I. Khan ; R. Kumar and M. Sastry(2003) "Extracellular biosynthesis of silver nanoparticles using the fungus Fusarium oxysporum". Colloids Surf B; 28: 313-318.

Becker, R.O. (1999) "Role of silver ions in destabilization of intermolecular adhesion forces measured by atomic force microscopy in Staphylococcus epidermidis biofilms" MetalBased Drugs, 6: 297-300.

Bindhu, M.R. and M. Umadevi (2013) "Synthesis of monodispersed silver nanoparticles using Hibiscus cannabinus leaf extract and its antimicrobial activity". Spectrochimica Acta Part A: Molecular and Biomolecular Spectroscopy.; 101:184-190.

Das, J.; M. Paul Das and P. Velusamy (2013) "Sesbania Grandiflora leaf extract mediated green synthesis of antibacterial silver nanoparticles against selected human pathogens" Spectrochim. Acta Part A: Mol. Biomol. Spec., 104: 265.

Duran, N.; P.D. Marcato ; O.L. Alves ; J.P.S. Da Silva ; G.I.H. De Souza ; F.A. Rodrigues and E. Esposito (2010) "Ecosystem protectection by effluent bioremediation: silver nanoparticles impregnation in a textile fabric process", J. Nanopart. Res., 12:285-292.

Durán, N. ; P.D. Marcato ; O.L. Alves ; G.I. Souza and E. Esposito (2005) "Mechanistic aspects of biosynthesis of silver nanoparticles by several Fusarium oxysporum strains". JoN; 3: 8 doi:10.1186/1477-3155-3-8 19.

Jiang, H.; S. Manolache ; A.C.L. Wong and F.S. Denes (2004) "Plasma-enhanced deposition of silver nanoparticles onto polymer and metal surfaces for the generation of antimicrobial characteristics", J. Appl. Polym., Sci., 93:1411-1422.

Liu, J.; S.Z. Qiao ; Q.H. Hu and G.Q. Lu (2001) "Magnetic Nanocomposites with Mesoporous Structures: Synthesis and Applications". Small, 7: 425-443.

Namvar, F.; H.S. Rahman ; R. Mohamad ; J. Baharara ; M. Mahdavi ; E. Amini ; M.S. Chartrand and S.K. Yeap (2014)." Cytotoxic effect of magnetic iron oxide nanoparticles synthesized via seaweed aqueous extract" .Int J Nanomedicine; 9: 2479-2488. 
Parida, U.K.; S.K. Biswal and B.K. Bindhani (2014). "Green Synthesis and Characterization of Gold Nanoparticles: Study of Its Biological Mechanism in Human SUDHL-4 Cell Line".Adv Biol Chem ; 4(6):360-375

Rai, M.; A. Yadav and A. Gade (2009) "Silver nanoparticles as a new generation of antimicrobials", Biotechnol. Adv., 27:76-83.

Salam, H.A. ; P. Rajiv ; M. Kamaraj ; P. Jagadeeswaran ; S. Gunalan and R. Sivaraj (2012) "Plants: Green Route for Nanoparticle Synthesis", Int. J. Biol. Sci., 1: 85-90.

Shameli, K. ; M.B. Ahmad ; A. Zamanian ; P. Sangpour ; P. Shabanzadeh ; Y. Abdollahi and M. Zargar (2012) "Green Biosynthesis of Silver Nanoparticles Using Curcuma Longa Tuber Powder". Int. J. Nanomedicine, 7: 5603-5610.

Tankhiwale, R. and S.K. Bajpai (2010)" Silver-nanoparticle-loaded chitosan lactate films with fair antibacterial properties"J. Appl. Polym. Sci., 115: 1894-1900.

Umoren, S.A.; I.B. Obot and Z. M. Gasem (2014). "Green Synthesis and Characterization of Silver Nanoparticles Using Red Apple (Malus domestica) Fruit Extract at Room Temperature", 5(3): 907-914.

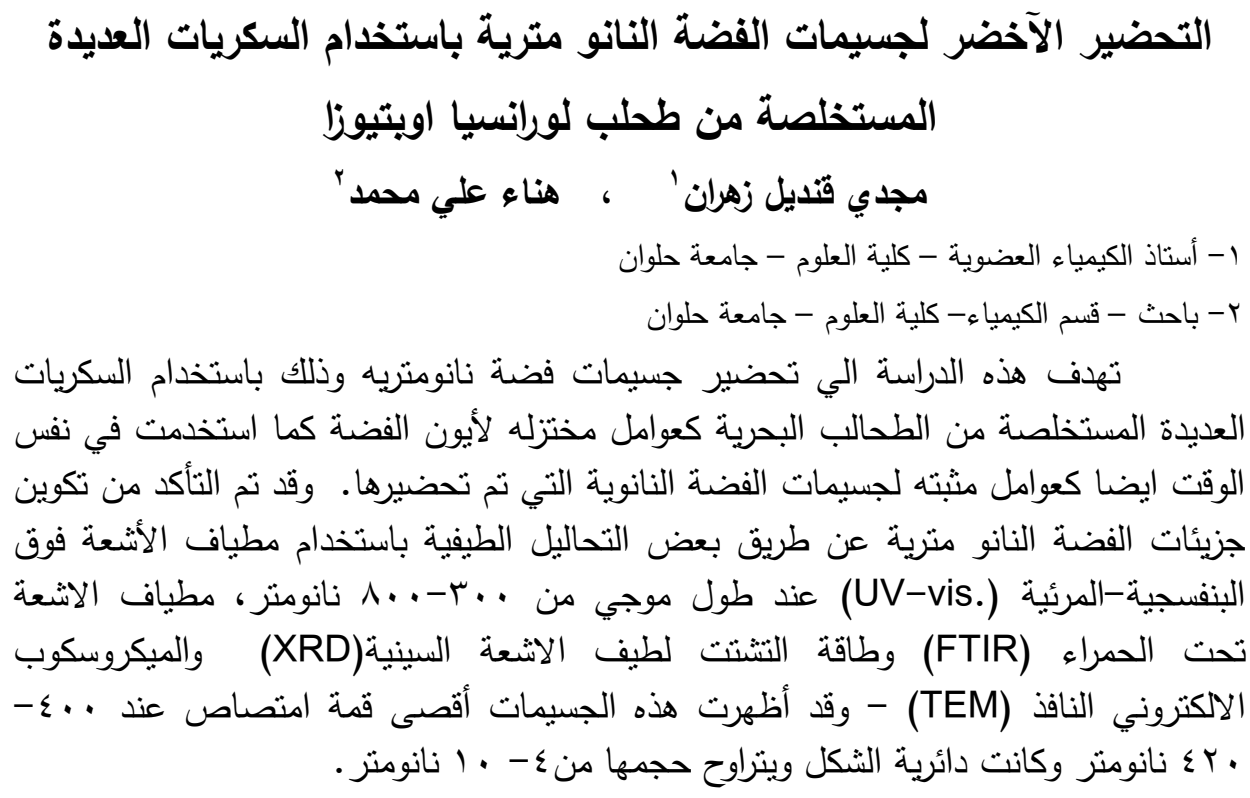

